American Journal of Infectious Diseases 8 (2): 70-72, 2012

ISSN 1553-6203

(C) 2012 Science Publications

\title{
Tuberculoma and Meningitis in a Young Girl: A Case Report
}

\author{
${ }^{1}$ Behroz Ahssan and ${ }^{2}$ Shahla Afrasiabian \\ ${ }^{1}$ Department of Neurology, \\ ${ }^{2}$ Department of Infectious Diseases, \\ Faculty of Medicine, Kurdistan University of Medical Sciences, Sanandaj, Iran
}

\begin{abstract}
Because of difficulty in early diagnosis and severe consequences of delay in diagnosis and treatment, tuberculosis meningitis is a serious problem for clinicians. Therefore recognizing rare cases of tuberculosis can improve clinician practice and save patients life in countries with high burden of disease. An 18-year-old girl suffered from fever, headache, illness, arthralgia, myalgia, constipation and weight loss for several weeks. In clinical exam, lethargy, Strabismus, neck stiffness, papilledema, bilateral cranial nerve VI palsy and left facial nerve palsy were observed. Pleocytosis in CSF and multiple small nodular enhancements was detected within cerebellum. The anti-tuberculosis plus corticosteroid were prescribed. Conclusion: In TB high burden regions such as Iran and Middle East, clinical diagnosis is an important tool for making decision about such cases. Though using corticosteroids in tuberculosis meningitis is still controversial, we recommend it strongly.
\end{abstract}

Key words: Intracranial tuberculoma, Tuberculosis, MRI

\section{INTRODUCTION}

Tuberculosis is a majour deseases in developing country. Every year about 2 million people die from Tuberculosis (TB) (WHO, 2009; Raviglione et al, 1995). The involvement of nervous system is detected in about 1\% of TB cases (Kuo et al., 2010; Rock et al., 2008). Signs and symptoms depend on organs involved in the disease. Central Nervous System (CNC) involvement is very important and life threatening. Tuberculous meningitis is a progressive disease and cranial nerve palsies may be resulted as well (Moon et al., 2008;. Because of difficulty in early diagnosis and severe consequences of delay in diagnosis and treatment, tuberculosis meningitis is a serious problem for clinicians (Christensen et al. 2011; Verdon et al., 1996). Moreover, tuberculoma is very rare and it can be mistaken with other diseases such as cancer metastases. Death from CNS involvement in TB is strongly associated with delays in diagnosis and treatment (Murthy, 2010). Therefore recognizing rare cases of tuberculosis can improve clinician practice and save patients life in countries with high burden of disease.

Case presentation: An 18-year-old girl from Iraq who suffered from fever, headache and vomiting was referred to Tohid hospital. She had fever, headache, illness, arthralgia, myalgia, constipation and vomiting during about 30 days earlier to her referral to clinic. Weight loss was 6 kilogram in 2 weeks. She had past records of two treatments in precedent 30 days, first as a typhoid case then as bacterial meningitis. But she was not cured and she got worse. 14 day after treatment, her eyes were goggled to medial side. Therefore she was referred to our hospital.

When she was admitted in our clinic, lethargy, Strabismus, neck stiffness, Axillary temperature of

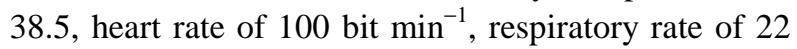
and blood pressure of $110 / 70 \mathrm{~mm} \mathrm{Hg}^{-1}$ were detected. Bilateral cranial nerve VI Palsy and left facial nerve palsy were seen. There was no any other nerve involvement and papilledema in fundoscopy exam.

The results of laboratory tests were reported as below:

- White Blood Cell (WBC): 8300 cell $\mu \mathrm{L}^{-1}$

- Hemoglobin: $14.7 \mathrm{gr} \mathrm{dL}^{-1}$

- Natrium (Na): 135

- Lactate Dehydrogenase (LDH): 528

- Cerebrospinal Fluid (CSF): Glucose: $104 \mathrm{mg} \mathrm{dL}^{-1}$, Protein: $270 \mathrm{mg} \mathrm{dL}^{-1}$, WBC: $435 \mathrm{~mm}^{3}$, Neutrophil: $70 \%$, Lymphocyte: $30 \%$, RBC: $230 \mathrm{~mm}^{3}$, Culture no growth

- Wright and Coombs Wright were negative

Corresponding Author: Shahla Afrasiabian, Department of Infectious Diseases, Faculty of Medicine,

Kurdistan University of Medical Sciences, Pasdaran Ave, Sanandaj, Iran Tel: +98-9181711493 


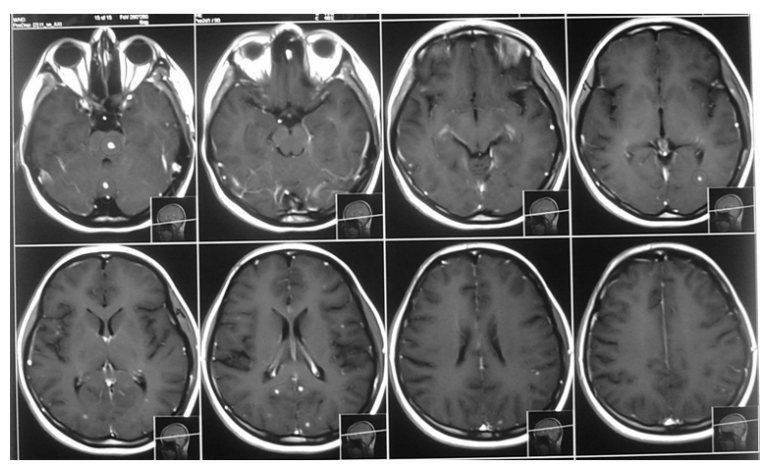

Fig. 1: Axial MRI of an 18-year old girl with tuberculoma and TB meningitis. The multiple nodular enhancements are seen within cerebellum, brain stem and both cerebral hemisphere subcortical white matter. (indicated in red cycles)

In chest Computed Tomography (CT) scan no evidence of abnormality was seen. Abdominal sonography was normal.

In brain Magnetic Resonance Imaging (MRI), multiple small nodular enhancement were seen within cerebellum, brain stem; both cerebral hemisphere subcortical white matter and mild peripheral edema around lesions were reported. Also lesions were centrally low signal in T2 Weighted granulomatous process (Fig. 1).

The clinical, laboratory and imaging evidences showed that she had tuberculoma plus TB meningitis. She was treated with isoniazid $300 \mathrm{mg}$ daily $^{-1}$, rifampin $600 \mathrm{mg}$ daily ${ }^{-1}$, pyrazinamide $25 \mathrm{mg} \mathrm{kg}^{-1}$, ethambutol $15 \mathrm{mg} \mathrm{kg}^{-1}$ for 2 month and isoniazid $300 \mathrm{mg}^{-1}$ daily $^{-1}$, rifampin $600 \mathrm{mg}$ daily $^{-1}$ and vitamin B6 $20 \mathrm{mg}$ daily $^{-1}$ for 8 month. Treatment was prescribed in first day according to history and clinical findings. In early period of treatment we ordered prednisolone $1 \mathrm{mg} \mathrm{kg}^{-1}$ for 30 days and it was tapered in 15 days. After 2 weeks of treatment initiation, she got better. The control brain MRI was clear.

\section{DISCUSSION}

Symptoms of CNS involvement by TB is similar to other diseases (Moon et al., 2008; Carlo, 2008). Fever, headache, weight loss, neuro-ophthalmic disorder (such as visual disorder) and meningismus, or some signs such as focal neurologic deficits (cranial nerve palsies, paresis, seizures) could be detected (Moon et al., 2008; Verdon et al., 1996). This may cause a delay in diagnosis and treatment.
First, this case was treated as a typhoid disease. Then she didn't cure and was treated as bacterial meningitis before referring to our clinic. Diagnosis of such cases can be problematic because of different signs and symptoms presented and it can resemble other diseases (Karstaedt et al., 1998).

Tuberculoma appears when tubercles enlarge in the brain parenchyma. It can be present in the absence or along with tuberculosis meningitis (Rock et al., 2008). However the exact diagnostic test for tuberculoma is biopsy, but it is not practical in CNS system. Most often, diagnosis depends on lumbar puncture and CSF examination in TB meningitis (Murthy, 2010).

CNS involvement could differ in any case. Hydrocephalus and basilar enhancement were present at the time of admission in 50 and $36 \%$ of patients, respectively (Karstaedt et al., 1998). The sixth cranial nerve is most commonly affected. Left VI nerve palsy was raised in our case due to intracranial pressure. Though bad prognostic features such as lethargy and facial palsy were seen in this case, the response to the treatment was very good. Maybe it was due to prescription of prednisolone along with anti-Tb drugs. The mortality rate is about $65 \%$ and it depends on treatment delay and expansion of lesions (Karstaedt et al., 1998; Verdon et al., 1996). Lee et al. (2002) recommended biopsy before starting anti tuberculous therapy, but we think clinical diagnosis is an important tool for making decision about such cases in TB high burden regions such as Iran and Middle East. Biopsy is not practical in most cases of tuberculoma.

Our case has cured by an appropriate treatment. Although using corticosteroids in tuberculosis meningitis is still controversial, we recommend it strongly.

\section{CONCLUSION}

In TB high burden regions such as Iran and Middle East, clinical diagnosis is a main tool for making decision about tuberculoma cases. Corticosteroids can rapidly decrease cerebral edema in meningitis and tuberculoma and it can prevent further permanent disabilities.

Consent: Written informed consent was obtained from the patients for publication of this study.

Competing interests: The authors declare that they have no competing interests.

Authors' contributions: All authors contributed to the writing of the manuscript. All authors read and approved the final manuscript. 


\section{REFERENCES}

Christensen, A.S.H., A.B. Andersen, V.O., Thomsen, P.H. Ande rsen and I.S. Johansen, 2011. Tuberculous meningitis in Denmark: A review of 50 cases. BMC Infect. Dis., 11: 47-47. DOI: 10.1186/1471-2334-11-47

Carlo, G., 2008. Raccolta Completa Delle Commedie Di Carlo Goldoni. 1st Edn., BiblioBazaar, ISBN10: 0559330499, pp: 352.

Karstaedt, A.S., N. Jones, M. Khoosal and H.H. CreweBrown, 1998. The bacteriology of pulmonary tuberculosis in a population with high human immunodeficiency virus seroprevalence. Int. J. Tuberc. Lung Dis., 2: 312-316. PMID: 9559402

Kuo, V.C., L.M. Sloan and M. Emmett, 2010. Central nervous system tuberculosis. Proc. Bayl. Univ. Med. Cent., 23: 359-360.

Lee, W.Y., K.Y. Pang and C.K. Wong, 2002. Brain tuberculoma in Hong Kong. Hong Kong Med. J., 8: 52-56. PMID: 11861995

Moon, S., J. Son and W. Chang, 2008. A case of oculomotor nerve palsy and choroidal tuberculous granuloma associated with tuberculous meningoencephalitis. Korean J. Ophthalmol., 22: 201-204. DOI: 10.3341/kjo.2008.22.3.201
Murthy, J.M., 2010. Tuberculous meningitis: The challenges. Neurol. India, 58: 716-722. DOI: 10.4103/0028-3886.72178

Raviglione, M.C., D.E. Snider and A. Kochi, 1995. Global epidemiology of tuberculosismorbidity and mortality of a worldwide epidemic. JAMA., 273: 220-226.

DOI: 10.1001/jama.1995.03520270054031

Rock, R.B., M. Olin, C.A. Baker, T.W. Molitor and P.K. Peterson, 2008. Central nervous system tuberculosis: Pathogenesis and clinical aspects. Clin Microbiol. Rev., 21: 243-261. DOI: 10.1128/ CMR.00042-07

Verdon, R., S. Chevret, J.P. Laissy and M. Wolff, 1996. Tuberculous meningitis in adults: review of 48 cases. Clin Infect. Dis., 22: 982-988. PMID: 8783697

WHO, 2009. Global tuberculosis control 2009: Epidemiology, Strategy and Financing. 1st Edn., World Health Organization, ISBN-10: 924156380X pp: 302. 